\title{
EGU2020-10936
}

https://doi.org/10.5194/egusphere-egu2020-10936

EGU General Assembly 2020

(c) Author(s) 2020. This work is distributed under

the Creative Commons Attribution 4.0 License.

\section{Monitoring and modeling the impact of soil conservation scenarios to limit overland flow and sediment supply from unpaved roads in a small catchment of Southern Brazil}

\author{
Alice Dambroz ${ }^{1}$, Jean Minella ${ }^{1}$, Cristiano Silva ${ }^{1}$, Alexandre Schlesner ${ }^{1}$, Felipe Bernardi ${ }^{1}$, Cláudia $^{2}$ \\ Barros $^{2}$, and Olivier Evrard ${ }^{3}$ \\ ${ }^{1}$ Soil Science Department, Federal University of Santa Maria (UFSM), Santa Maria, Brazil (jean.minella@ufsm.br) \\ ${ }^{2}$ Soil Science Department, Federal University of Rio Grande do Sul (UFRGS), Porto Alegre, Brazil \\ ${ }^{3}$ Laboratoire des Sciences du Climat et de l'Environnement (LSCE/IPSL), Unité Mixte de Recherche 8212 (CEA/CNRS/UVSQ), \\ Université Paris-Saclay, Gif-sur-Yvette, France
}

Erosion processes are accelerated by the presence of unpaved roads in catchments with shallow soils and steep slopes, favoring overland flow and sediment connectivity between hillslopes and the river network. Soil erosion modeling studies conducted at the catchment scale focus primarily on the hydrological behavior of cultivated hillslopes. Few studies address unpaved roads and suitable practices to limit their impact on hydro-sedimentary transfers in a catchment system. This study simulates soil conservation measures on unpaved roads and hillslopes and their effect on the hydrological and erosive dynamics in a small order catchment. The rainfall events were monitored at Lajeado Ferreira's creek, in Arvorezinha, Southern Brazil $\left(1.2 \mathrm{~km}^{2}\right)$. The catchment is characterized by shallow soils, steep slopes, intense agricultural activity and sediment yields (SY) of around $150 \mathrm{t} \mathrm{km}^{-2} \mathrm{y}^{-1}$. Unpaved roads cover about 3\% of the catchment surface area and supply $36 \%$ of the annual average SY. The Limburg Soil Erosion Model (LISEM) was used to simulate the roads' impact on soil erosion. Eight rainfall events, monitored from 2014 to 2017, were calibrated. Rainfall ranged from 9 to $97 \mathrm{~mm}$, total runoff volume (Q) varied from 1462 to $60765 \mathrm{~m}^{3}$ and SY from 0.6 to 81 tons. These events represent different precipitation and land use conditions, so that the road's effects on the hydrological dynamics of this catchment may be investigated. Then, modeling scenarios consisting of three levels of intervention to mitigate sediment supply were tested. The low intervention (LI) level was based on cost-efficient practices, applied to the road only through the installation of rockfill and energy-deflecting small reservoirs. The medium intervention (MI) included the sowing of grass for gutter protection on the road area and filter strips were installed near the drainage channels. For the high intervention scenario ( $\mathrm{HI})$, additional grass strips were installed on hillslopes. Their impact was evaluated by comparing the hydrosedimentological variables $\mathrm{Q}\left(\mathrm{m}^{3}\right)$, peak flow $\left(\mathrm{L} \mathrm{s}^{-1}\right)$ and $\mathrm{SY}$ (tons), modelled at the catchment's outlet. Rockfill and energy-deflecting small reservoirs were not enough for reducing peak flow (Qp), on average. Indeed, the main proportion of overland flow originates from other landscape components, such as hillslopes. Under the MI and HI scenarios, Qp decreased by 2 and $46 \%$, respectively. The $\mathrm{LI}$ and $\mathrm{MI}$ scenarios led to an average Q reduction of $12 \%$, compared to $53 \%$ 
under scenario $\mathrm{HI}$. For one event, $\mathrm{HI}$ promoted a reduction of $92 \%$ of calculated $\mathrm{Q}$, representing $15,693 \mathrm{~m}^{3}$. HI also showed the most positive effects on limiting SY. It becomes evident that hillslope interventions are necessary, as they allow increasing infiltration, reducing both runoff volume and stream power when the flow reaches the roads. For rainfall events of higher magnitude, it was observed that HI was responsible for reducing Qp between 9 and 25\%, while during smaller events, this reduction reached 61 to 93\%. This indicates the importance of managing roads in order to reduce runoff energy and concentration, but also to take measures on hillslopes to limit overland flow and erosion inputs, as well as to delay peak Q. 